\title{
Critical Torque for Kink Formation in Double-Stranded DNA
}

\author{
Hao Qu, Yong Wang, Chiao-Yu Tseng, and Giovanni Zocchi* \\ Department of Physics and Astronomy, University of California Los Angeles, Los Angeles, California 90095-1547, USA
}

(Received 11 May 2011; published 11 November 2011)

\begin{abstract}
We measure the bending energy of double-stranded DNA in the nonlinear (sharply bent) regime. The measurements are obtained from the melting curves of stressed DNA ring molecules. The nonlinear elastic behavior is captured by a single parameter: the critical torque $\tau_{\mathrm{c}}$ at which the molecule develops a kink. In this regime, the elastic energy is linear in the kink angle. This phenomenology is the same as for the previously reported case of nicked DNA. For the sequences examined, we find $\tau_{\mathrm{c}}=31 \mathrm{pN} \times \mathrm{nm}$. This critical torque corresponds to a characteristic energy scale $(\pi / 2) \tau_{\mathrm{c}}=12 k T_{\text {room }}$ relevant for molecular biology processes associated with DNA bending.
\end{abstract}

DOI: 10.1103/PhysRevX.1.021008

Subject Areas: Biological Physics, Nonlinear Dynamics, Soft Matter

\section{INTRODUCTION}

The elastic energy of long polymers has been discussed since the dawn of polymer physics [1], but compact polymeric molecules such as globular proteins, ribozymes, and short DNA molecules are likewise deformable. This aspect is often approached in structural terms, leading to the fundamental concepts of induced fit and allostery [2], while less often discussed are the corresponding stresses and elastic energies, although some of the early work was in this direction $[3,4]$. More recently, the elastic energy of molecular deformations has been invoked as the basis for conformational proofreading mechanisms [5]. Namely, the selectivity of ligand binding through the induced-fit mechanism may be enhanced by the elastic energy of the protein's deformation. From a materials-science perspective, it is in the stress-strain relations rather than the structural description of conformational motion that one may expect a degree of universality to emerge. We are specifically interested in the mechanical response of short $(<60 \mathrm{bp})$ DNA molecules forced to bend substantially, for two main reasons. First we use these highly bent DNAs as molecular springs in protein-DNA chimeras [6,7]. The use of DNA oligomers in highly bent configurations to generate piconewton forces at nanometer $(\mathrm{nm})$ scales requires a quantitative understanding of DNA mechanics under such conditions. Second, the bending elasticity of doublestranded (ds) DNA at short length scales plays a crucial role in natural biological systems. For example, in the cases of viral genome packaging, eukaryotic nucleosome compaction, and many transcription regulation steps, the DNA is sharply bent over a range of tens to hundreds of base pairs. DNA bending therefore has a strong influence on the energy scales of many biological processes $[8,9]$.

\footnotetext{
*zocchi@physics.ucla.edu
}

Published by the American Physical Society under the terms of the Creative Commons Attribution 3.0 License. Further distribution of this work must maintain attribution to the author(s) and the published article's title, journal citation, and DOI.
Quantitative knowledge of DNA linear bending elasticity comes from single-molecule force-extension experiments on $\sim 10 \mathrm{~kb}$-long DNA [10], where kb stands for kilo-base pair. These measurements are well described by the wormlike-chain (WLC) model with one microscopic parameter, the persistence length $l_{\mathrm{p}} \approx 50 \mathrm{~nm}$ (corresponding to $\sim 150 \mathrm{bp}$ ). The bending energy used in the WLC model is that of a thin rod in the linear-elasticity regime:

$$
E_{\mathrm{WLC}}=\int_{0}^{2 L} d s \frac{1}{2} \frac{B}{R^{2}(s)},
$$

where $R$ is the radius of curvature, $s$ the arc length along the rod, $2 L$ the contour length, and $B=k T l_{\mathrm{p}}$ the bending modulus $\left(B \approx 200 \mathrm{pN} \times \mathrm{nm}^{2}\right)$. This form of the energy must break down for $R$ sufficiently small, but this is not seen in the DNA stretching experiments where the highcurvature configurations are irrelevant [11]. In contrast, cyclization experiments initially suggested [12] that the high-curvature states of DNA are much more flexible than predicted by (1). There is some controversy about the exact range of validity of (1). If we write this range of validity as $R>C l_{\mathrm{p}} /(2 \pi)$, where $C$ is a numerical factor (of order 1), then the initial suggestion from cyclization experiments [12] was $C \approx 0.7$, whereas Du et al. argued that $C$ is lower [13]; using DNA minicircles the study [14] found that $C \approx 0.5$. Other studies also reported softening of the DNA at small scales [15]; in particular, the Atomic Force Microscopy (AFM) study [11] reported that the measured correlation functions are best described by a linear (rather than quadratic) dependence of the energy on the bending angle. The structural transition responsible for this softening is likely to involve formation of a small bubble (single-stranded or ss region) in the DNA, as pointed out by Yan and Marko [16].

Cyclization experiments [12] and AFM imaging experiments [11] rely on thermal fluctuations to realize the highcurvature states; one difficulty is then that the probability of these states is small. In our approach, the high-curvature states are designed into a mechanically constrained 
molecule [17], and the elastic energy is measured by thermodynamics methods $[18,19]$. Using the construction of Fig. 1, we were able to obtain a complete characterization of the bending energy from the linear to the nonlinear (high bending) regime, for the case of nicked ds DNA $[19,20]$. Here we characterize the bending energy for the case of non-nicked (i.e. intact) DNA. This required a different measurement method, based on melting-curve analysis. Namely, as the temperature is raised, the DNA double helix "melts" into two single strands. Because ds and ss DNA have different UV absorption coefficients, the transition can be easily characterized spectroscopically in the form of a plot of UV absorption of the sample vs temperature. This is called a "melting curve." The elastic energy in the stressed DNA shifts this melting curve to lower values of the temperature. We first summarize our previous results for nicked DNA, as they are relevant to the present work.

Our previous study was performed on slightly different molecules, consisting of two linear strands (as opposed to one linear and one circular strand), also hybridized as in Fig. 1. These molecules effectively contain a nick at the center of the ds part (i.e., in the drawing of Fig. 1, the backbone of the blue strand is broken at one site). In this situation, two such molecules can form a dimer (a polymer formed from two molecules of a monomer) in which the elastic energy is relaxed. Base pairing in one dimer is identical to base pairing in two monomers [Fig. 2(a)], and the elastic energy, $E_{\mathrm{el}}$, of the monomer can be extracted from the monomer-dimer equilibrium according to

$$
E_{\mathrm{el}}=\frac{1}{2} k T \ln \frac{X_{D}}{X_{M}^{2}},
$$

where $X_{M}, X_{D}$ are the mole fractions of monomers and dimers at equilibrium. (A small correction is further

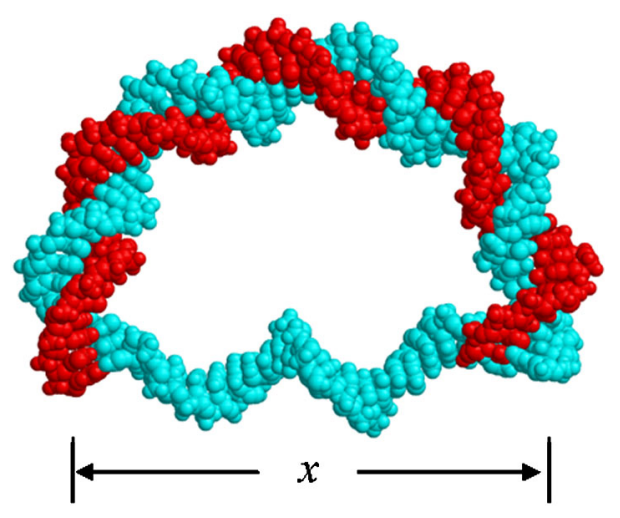

FIG. 1. Cartoon of a stressed DNA molecule used in the experiments. The ds DNA is from the Protein Data Bank (PDB) structure $1 \mathrm{KX} 5$, and the ss DNA is from PDB 1BNA. The molecule is formed by partially hybridizing a ss DNA loop (blue) with a linear complementary strand (red). The elastic energy of this molecule consists of the bending energy of the ds part and the stretching energy of the ss part. (a)
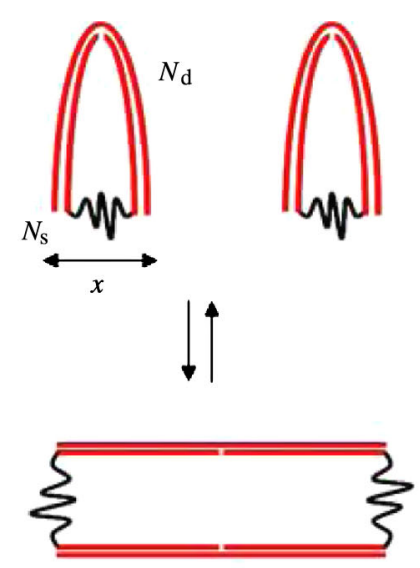

(b)

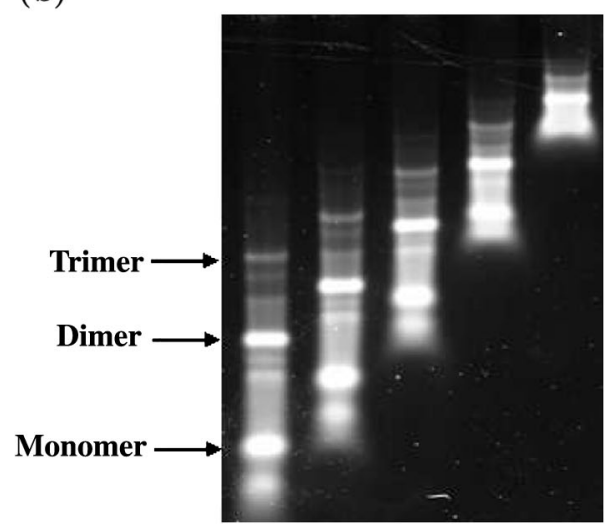

FIG. 2. (a) Sketch of the monomer-dimer equilibrium used to measure the elastic energy of the monomer in [20]. The monomer is stressed because the ds part of the molecule has to bend and the ss part has to stretch, but the stress is relaxed in the dimer. (b) Example of a gel used to determine the concentrations of monomers and dimers in the dimerization equilibrium experiments [20]. All lanes were loaded with the same sample, at successive $(10 \mathrm{~min})$ intervals. The purpose is to extrapolate back in time the initial (equilibrium) concentrations of the two species at "zero" time, since some monomer-dimer interconversion occurs in the gel. This interconversion produces the "smear" and extra bands visible mostly between the monomer and dimer bands; it is accounted for by the reaction-diffusion model we use to fit the intensity profiles and extrapolate the concentrations to zero time. This method is detailed in [19]. The band in front of the monomer band is ss DNA and arises because the stoichiometry of the two strands used for the monomer construction is not exactly $1: 1$.

applied to this energy to account for stretching of the ss part in the dimer; this correction is described in [19].) $X_{M}$, $X_{D}$ are determined by gel electrophoresis of the equilibrated samples [Fig. 2(b)]. We performed measurements of $E_{\mathrm{el}}$ for varying $N_{\mathrm{s}}$ (the number of bases in the ss part of the molecule) and fixed $N_{\mathrm{d}}$ (the number of bp in the ds part), which amounts to changing the end-to-end distance (EED) $x$ of the bent ds part. For increasing $N_{\mathrm{s}}$, the ss spring in Fig. 1 becomes softer, the EED $x$ increases, and one obtains 
the energy curve shown in Fig. 3(a) (reproduced from [20]). This energy is the sum of the elastic energy of the bent ds DNA and the stretched ss DNA: $E_{\text {tot }}=E_{\mathrm{d}}+E_{\mathrm{s}}$. The break or transition in the energy curve corresponds to formation of a kink in the ds DNA: to the right of the transition [in Fig. 3(a)], the ds DNA is smoothly bent; to

(a)

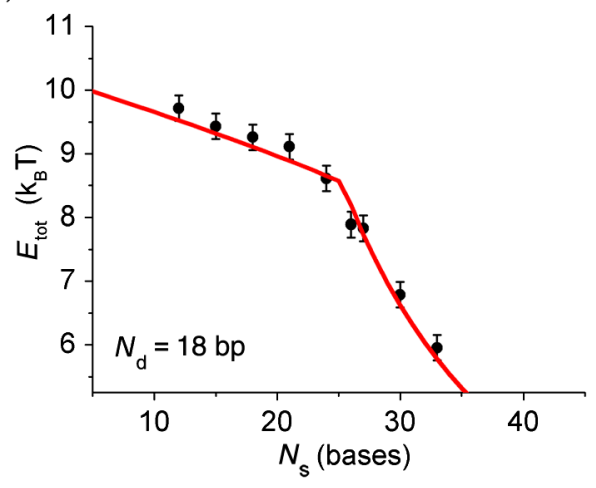

(b)

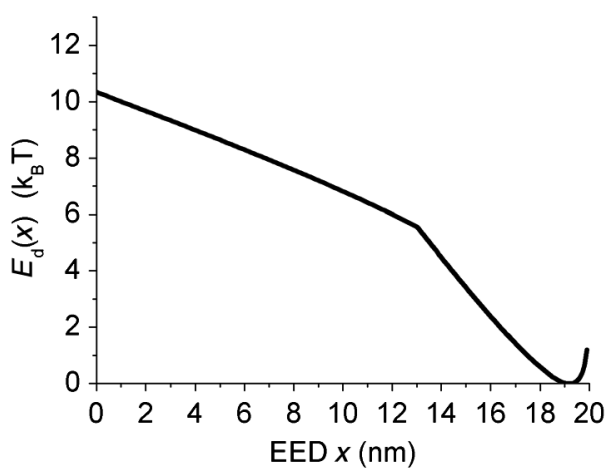

(c)

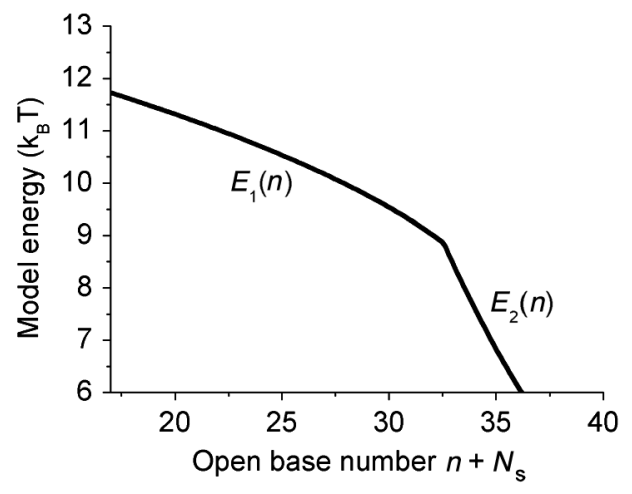

FIG. 3. (a) The total elastic energy $E_{\text {tot }}$ of the nicked molecules measured using the dimerization equilibrium method vs $N_{\mathrm{s}}$, the number of bases in the ss part. The graph shown is for $N_{\mathrm{d}}=18$ (the number of bases in the ds part). The solid line is calculated using Eq. (3) for $E_{\mathrm{d}}(x)$ with $L=3 \mathrm{~nm}, \tau_{\mathrm{c}}=27.0 \mathrm{pN} \times \mathrm{nm}$, $B=50 \mathrm{kT} \times \mathrm{nm}$, and $l_{\mathrm{s}}=0.764 \mathrm{~nm}$. (b) Plot of the ds DNA bending energy $E_{\mathrm{d}}(x)$ vs EED $x$ from Eq. (3) for $N_{\mathrm{d}}=60$ (contour length $2 L=20 \mathrm{~nm}$ ), using $\tau_{\mathrm{c}}=27.0 \mathrm{pN} \times \mathrm{nm}$. (c) The graph of the energy function used in the modified zipper model from Eqs. (8) and (9) as a function of open bases $n+N_{\mathrm{s}}$. the left it is kinked. This behavior is captured (solid line in Fig. 3(a)] by introducing one microscopic parameter (in addition to the bending modulus $B$ ), namely, a critical torque $\tau_{\mathrm{c}}$ at which the ds DNA develops a kink [20]. This torque couples to DNA bending, not twist, i.e., the torque in question is perpendicular to the helix axis (not parallel to it). For a quantitative description, we constructed a model where the ds DNA, under increasing forces applied at the ends, bends smoothly with the thin rod-bending energy (1) until a critical internal torque $\tau_{\mathrm{c}}$ is reached. (For zero torque boundary conditions at the ends, this happens in the middle of the rod.) From then on, the rod is kinked, and the torque at the kink is constant, equal to $\tau_{\mathrm{c}}$ (i.e., the elastic energy is linear in the kink angle). We obtained an approximate analytic expression for the elastic energy of this model as a function of the EED $x$ of the DNA [20]:

$E_{\mathrm{d}}(x)= \begin{cases}\tau_{\mathrm{c}} \arccos \frac{x}{2 R} & \text { for } 0<x<x_{\mathrm{c}} \\ \frac{5 B}{L} \frac{x_{0}-x}{2 L}-T \ln \left(\frac{2 L-x}{2 L-x_{0}}\right) & \text { for } x_{\mathrm{c}}<x<x_{0},\end{cases}$

where $\quad R=L\left(1-2 \gamma^{2} / 45\right), \quad \gamma=L \tau_{\mathrm{c}} /(2 B), \quad x_{0}=$ $2 L(1-L T /(5 B))$, and the contour length of the DNA is $2 L=0.33 \mathrm{~nm} \times N_{\mathrm{d}}$. The upper form corresponds to the kinked solution, the lower to the smoothly bent one. The critical EED $x_{\mathrm{c}}$ is found by equating the upper and lower expressions. Here and throughout the rest of the paper, we use units where the Boltzmann constant $k=1$.

This energy is plotted in Fig. 3(b) for the case of a DNA 60 mer $(2 L=20 \mathrm{~nm})$, using $\tau_{\mathrm{c}}=27.0 \mathrm{pN} \times \mathrm{nm}$, the result for nicked DNA [20]. Using the energy (3) and the Marko-Siggia expression [21] for the stretching elastic energy $E_{\mathrm{s}}(x)$ of the ss DNA, we calculate the total elastic energy of the molecules of Fig. 1:

$$
E_{\mathrm{tot}}=E_{\mathrm{d}}\left(x_{\mathrm{eq}}\right)+E_{\mathrm{s}}\left(x_{\mathrm{eq}}\right) \text {, }
$$

where $x_{\mathrm{eq}}$ is calculated from the mechanical equilibrium condition $\left(\partial E_{\mathrm{d}} / \partial x\right)_{x_{\mathrm{eq}}}+\left(\partial E_{\mathrm{s}} / \partial x\right)_{x_{\mathrm{eq}}}=0$. The solid line in Fig. 3(a) represents $E_{\text {tot }}$ above, and is seen to fit the data well. From the fit, we obtain the value of the critical torque, $\tau_{\mathrm{c}}=27.0 \mathrm{pN} \times \mathrm{nm}$, for this case (nicked DNA) [20].

\section{MATERIALS AND METHODS}

\section{A. Sample preparation}

The synthetic DNA oligomers were bought from Integrated DNA Technology, phosphorylated at the 5' end. The oligomers were then ligated by T4 DNA ligase (from New England Biolabs) to form a single-stranded (ss) loop, with the help of a $12 \mathrm{mer}$ or $18 \mathrm{mer}$ splint (from Integrated DNA Technology) complementary to the sequence around the ligation position. The splint and any other linear DNA components were removed by restriction enzymes Exonuclease I and Exonuclease III (from New England Biolabs), which the circular DNA is immune to. 
After that, the circular products passed through an HPLC Uno Q1 ion exchange column (from Bio-rad) in order to separate single-loop (product of intramolecular ligation) and double-loop (product of intermolecular ligation) molecules. The single-loop fraction was collected, annealed (at $\sim 0.065^{\circ} \mathrm{C} / \mathrm{min}$ ) with an equimolar amount of partially complementary strands and diluted to a final concentration $0.1 \mu \mathrm{M}$ for the melting-curve measurements. The samples were prepared in a $10 \mathrm{mM}$ Tris buffer with $1 \mathrm{mM}$ ethylenediaminetetraacetic acid (EDTA), $100 \mathrm{mM} \mathrm{NaCl}$, and $5 \mathrm{mM} \mathrm{MgCl}_{2}$, and $\mathrm{pH}=7.9$.

\section{B. Melting-curve measurements}

UV absorption measurements at $260 \mathrm{~nm}$ were carried out on a Beckman Coulter DU 640 spectrophotometer at a controlled temperature from $25^{\circ} \mathrm{C}$ to $90^{\circ} \mathrm{C}$. The heating rate was $0.2^{\circ} \mathrm{C} / \mathrm{min}$, and the reading interval was $0.5^{\circ} \mathrm{C}$. During the measurements, samples were kept in quartz cuvettes, which were sealed by polytetrafluoroethylene (PTFE) stoppers to prevent evaporation.

\section{DNA sequences}

For $N_{\text {loop }}=45$, the loop sequence (from the 5 , ligation end) is: Cloop45: CACACGTGAGAGCAGCAGGCAAT GACAGTAGACATACGACGACTC. The corresponding complementary sequences are (the numbers in the parentheses are the lengths of the ds and ss tracts in the molecule which results from hybridizing with the loop sequence): D30C45 $\left(N_{\mathrm{d}}=30, N_{\mathrm{s}}=15\right):$ CTGCTCTCACGTGTG GAGTCGTCGTATGTC. D31C45 $\left(N_{\mathrm{d}}=31, N_{\mathrm{s}}=14\right)$ : GCTGCTCTCACGTGTGGAGTCGTCGTATGTC. D32C45 $\left(N_{\mathrm{d}}=32, N_{\mathrm{s}}=13\right)$ : TGCTGCTCTCACGTGTG GAGTCGTCGTATGTC. D33C45 $\left(N_{\mathrm{d}}=33, N_{\mathrm{s}}=12\right)$ : CTGCTGCTCTCACGTGTGGAGTCGTCGTATGTC. For $N_{\text {loop }}=60$, the loop sequence is: Cloop60: CAC ACGTGAGAGCAGCAGCAGGCAATCGATACACACA CAGTAGACGACATACGACGACTC. The complementary sequences are: $\mathrm{D} 35 \mathrm{C} 60 \quad\left(N_{\mathrm{d}}=35, \quad N_{\mathrm{s}}=25\right)$ : GCTGCTGCTCTCACGTGTGGAGTCGTCGTATGTCG. D37C60 $\left(N_{\mathrm{d}}=37, N_{\mathrm{s}}=23\right)$ : TGCTGCTGCTCTCACG TGTGGAGTCGTCGTATGTCGT. D39C60 $\left(N_{\mathrm{d}}=39\right.$, $\left.N_{\mathrm{s}}=21\right)$ : CTGCTGCTGCTCTCACGTGTGGAGTCGT CGTATGTCGTC. D41C60 $\left(N_{\mathrm{d}}=41, \quad N_{\mathrm{s}}=19\right)$ : CC TGCTGCTGCTCTCACGTGTGGAGTCGTCGTATGTC GTCT. D43C60 $\left(N_{\mathrm{d}}=43, \quad N_{\mathrm{s}}=17\right)$ : GCCTGCTG CTGCTCTCACGTGTGGAGTCGTCGTATGTCGTCTA. D45C60 $\quad\left(N_{\mathrm{d}}=45, \quad N_{\mathrm{s}}=15\right): \quad$ TGCCTGCTGCTG CTCTCACGTGTGGAGTCGTCGTATGTCGTCTAC.

\section{RESULTS}

The purpose of this study is to investigate whether the quantitative description in terms of a critical torque $\tau_{\mathrm{c}}$ given in [20] and summarized in the Introduction is still valid in the absence of the nick (i.e., for intact DNA), and if so, what is the value of $\tau_{\mathrm{c}}$ for intact DNA. We use the molecular construction of Fig. 1, now without nick, i.e., the blue strand is circular and the red strand is linear. Measuring the elastic energy based on the monomer-dimer equilibrium is not practical in this case because dimer formation would now involve a large "defect" in the structure of the base pairing; this defect suppresses the dimers and also introduces a large unknown term in the free energy. Instead, we examine the thermal melting curve of the molecule of Fig. 1. Compared to the corresponding linear molecule (obtained by cutting the circular strand of the molecule of Fig. 1 in the middle of the ss part, i.e., into a linear molecule that has an identical base sequence as the circular molecule but no built-in stress), the melting transition of the circular molecule is shifted to lower temperatures because of the destabilizing effect of the elastic energy. This is a large effect (Fig. 4), and we use it to determine the elastic energy of the circular molecule.

\section{A. Experimental melting curves}

In more detail, we will compare three different melting curves, obtained for the circular molecule, the corresponding linear molecule, and the nicked molecule (this last obtained by cutting the circular strand of the molecule of Fig. 1 in the middle of the ds part). The nicked molecule will be used as a control for our melting-curve analysis, based on the independent measurements of the elastic energy with the dimerization equilibrium method. Figure 4 shows two sets of melting curves, each set corresponding to one of the data points of Fig. 5. The melting curves, obtained by UV absorption spectroscopy, are normalized in the interval $[0,1]$ to reflect the fraction of open bp. Each melting curve represents one experimental run (not the average of several runs); occasionally, a melting curve was repeated, with essentially identical result. For each set of three melting curves corresponding to the same sequence (circular molecule, nicked molecule, linear molecule), we normalized the experimental melting profiles (by a vertical shift and dilation) as follows.

(1) Even at temperatures beyond the strand-separation temperature, the UV absorption continues to increase, as is well known, due to unstacking in the single strands [22]. This high-temperature (stranddissociated) part of the three curves is made to overlap. (Since the sequences are the same, we expect the ss unstacking transition to be the same.)

(2) There is typically a break in the melting curve at the strand-dissociation temperature [22]; the corresponding value of the ordinate is set to 1 .

(3) The low-temperature plateaus $(300 \mathrm{~K}<T<$ $320 \mathrm{~K}$ ) are set to 0 for the linear and the nicked molecules, while for the circular molecule, the lowtemperature region is set to a value corresponding to 3 open bp. The measurements show that the ds part of these molecules is kinked, and this normalization 


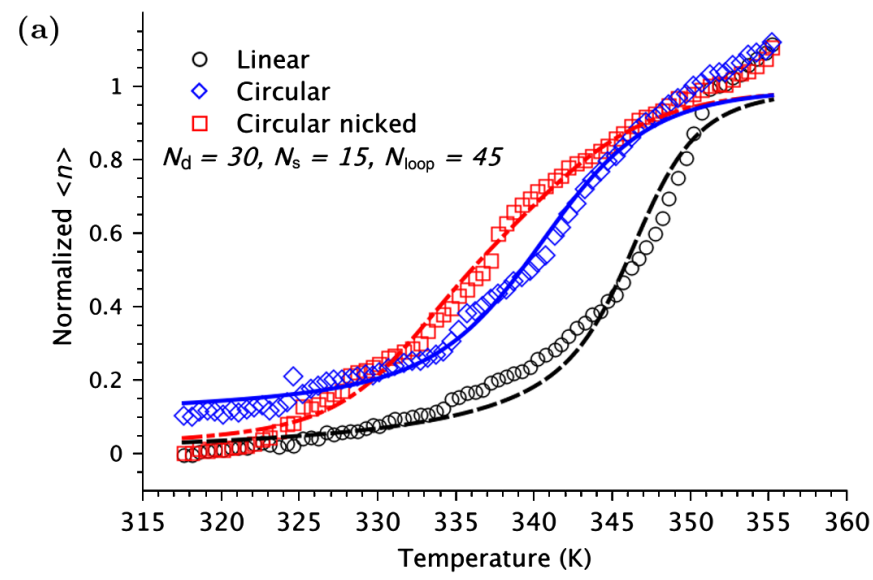

(b)

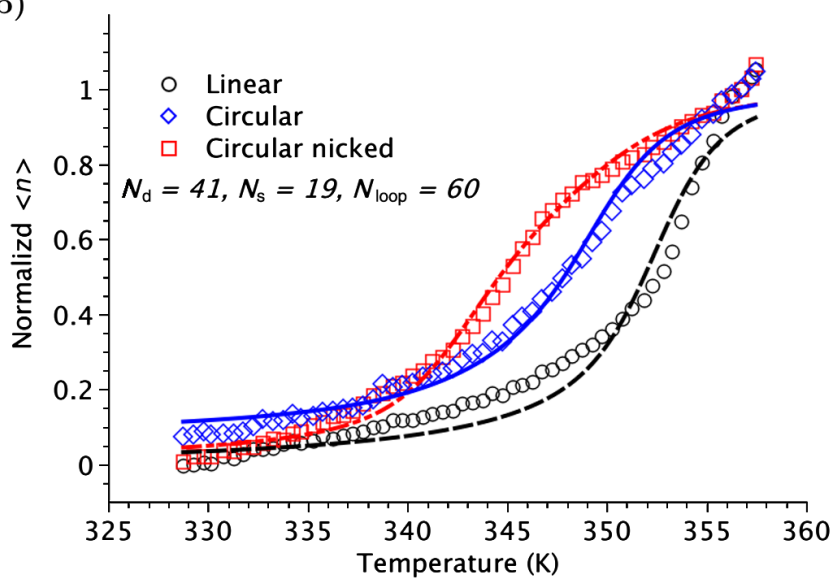

FIG. 4. Experimental melting curves and the corresponding fits (dashed lines) used for measurements of the elastic energy of the molecules of Fig. 1. The data are UV-absorption profiles at $260 \mathrm{~nm}$ and are normalized as described in the text. We show two sets: (a) $N_{\mathrm{d}}=30, N_{\mathrm{s}}=15, N_{\text {loop }}=45$ and (b) $N_{\mathrm{d}}=41$, $N_{\mathrm{s}}=19, N_{\text {loop }}=60$. Circles indicate linear molecules; diamonds, circular molecules; squares, circular nicked molecules. The curves are fits using Eqs. (10)-(12), as described in the text. The destabilizing effect of the elastic energy is evident in the large shift of the melting profiles of the circular molecules.

reflects the likely presence of a $\sim 3$ bp bubble at the kink [16]. This normalization improves the quality of the fits, which we describe next, and is thus, to some extent, suggested by the experimental data themselves.

\section{B. Model}

To extract the elastic energy of the circular molecule from the melting curves we of course need a model. The main consideration is to be able to fit all the melting curves with a small number of parameters. This is not necessarily simple because the melting profiles of the stressed molecules have more structure in them than simple S-shaped curves. For example, a two-states model proves inadequate
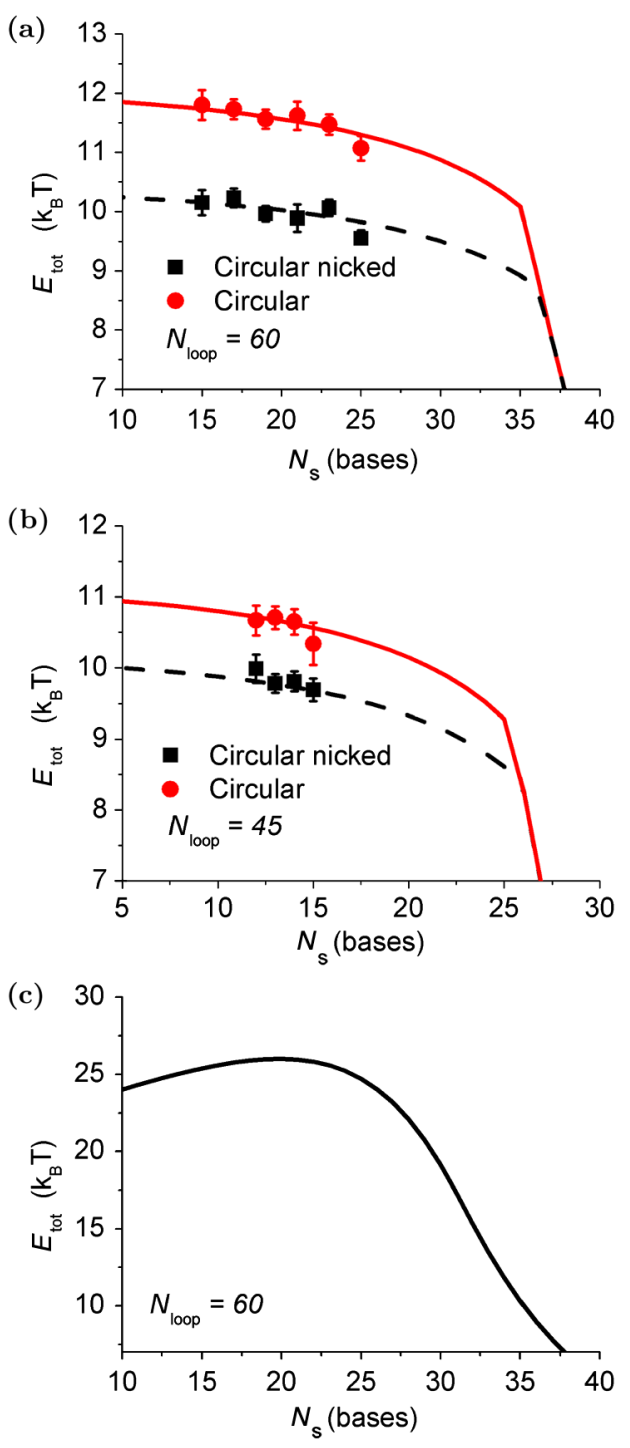

FIG. 5. The total elastic energy $E_{\text {tot }}$ of the molecules of Fig. 1, measured from the melting-curve analysis. The measurements are displayed vs $N_{\mathrm{s}}$ (the number of bases in the ss part of the molecule) at fixed $N_{\text {loop }}=N_{\mathrm{s}}+N_{\mathrm{d}}$ (where $N_{\mathrm{d}}=$ number of bases in the ds part of the molecule), for (a) $N_{\text {loop }}=60$ and (b) $N_{\text {loop }}=45$. Squares indicate nicked molecules; circles, intact molecules. Error bars reflect the statistical error of the fits of the melting curves. The lines are calculated using Eq. (3) for the bending energy of ds DNA, as explained in the text, with these parameter values: (a) $\tau_{\mathrm{c}}=27.0 \mathrm{pN} \times \mathrm{nm}$ for the nicked molecules (dashed line) and $\tau_{c}=31.3 \mathrm{pN} \times \mathrm{nm}$ for the intact molecule (solid line); (b) $\tau_{\mathrm{c}}=26.3 \mathrm{pN} \times \mathrm{nm}$ for the nicked case and $\tau_{c}=28.8 \mathrm{pN} \times \mathrm{nm}$ for the intact case; and identical values for the other parameters: $B=50 \mathrm{kT} \times \mathrm{nm}, l_{\mathrm{s}}=$ $0.8 \mathrm{~nm} . \tau_{\mathrm{c}}$ is the only adjustable parameter, and it determines both the level and the slope of the roughly linear behavior of $E_{\text {tot }}$ vs $N_{\mathrm{s}}$ in the regime shown, which is characterized by a kink in the ds part of the molecule. (c) The total elastic energy $E_{\text {tot }}$ calculated for the case $N_{\text {loop }}=60$ using the WLC energy in Eq. (1) instead of the expression in Eq. (3). More precisely, we used the approximation in Eq. (3) (lower branch) to the exact WLC energy, for ease of computation. 
in this case. Even for the linear molecules, the nearestneighbor model as implemented in the public domain servers [23], although predicting quite well the midpoint of the transition, fails to reproduce the shape of the melting curve.

We use a zipper model [24] modified to include the elastic energy term. In the zipper model, the oligonucleotide melts from the ends; the states of the system are parametrized by the number of open bp $n$, and there is a fixed energy cost $\epsilon$ and entropy gain $\sigma$ of opening a bp. These parameters represent average values for the particular sequence. One could of course introduce base pair dependent parameters in the zipper model, but a fixed set of such parameters does not represent different melting curves well (and the same applies to the nearest-neighbor model) [25]. Other models such as the Peyrard-BishopDauxois model do better in this respect $[25,26]$, but it is not obvious how to couple the elastic energy to them. In the interest of keeping the number of fitting parameters to a minimum, we therefore choose average $\epsilon$ and $\sigma$ in the zipper model.

Next we need an expression for the elastic energy $E(n)$ of the state characterized by $n$ (the number of open bp). We use two branches for this energy function because, as $n$ increases, the number of base pairs in the ds part of the molecule decreases $\left(N_{\mathrm{d}} \rightarrow N_{\mathrm{d}}-n\right.$; see Fig. 1$)$, while the number of bases in the stretched ss part of the molecule increases $\left(N_{\mathrm{s}} \rightarrow N_{\mathrm{s}}+n\right)$, so at some point the molecule will make a transition from the kinked to the smoothly bent state [see Fig. 3(a)]. We therefore write the partition sum for this model as:

$$
\begin{aligned}
Z= & \sum_{n=0}^{n_{0}}(n+1) \exp \left[-\frac{n(\epsilon-\sigma T)}{T}\right] \exp \left(-\frac{E_{1}(n)}{T}\right) \\
& +\sum_{n=0}^{N_{\mathrm{d}}-1}(n+1) \exp \left[-\frac{n(\epsilon-\sigma T)}{T}\right] \exp \left(-\frac{E_{2}(n)}{T}\right)+Z_{N},
\end{aligned}
$$

where $\epsilon$ and $\sigma$ are the average energy cost and entropy gain (for this particular sequence) of opening one bp, $E_{1}(n)$ is the elastic energy in the kinked state, $E_{2}(n)$ the elastic energy in the smoothly bent state, and $n_{0}$ the value of $n$ at which the molecule transitions from kinked to smoothly bent. The factors $(n+1)$ correspond to allowing the molecule to melt from both ends, and $Z_{N}$ is the statistical weight of the dissociated state:

$$
Z_{N}=\exp \left[-\frac{N_{\mathrm{d}}(\epsilon-\sigma T)}{T}\right] \exp \left(\sigma_{\mathrm{d}}\right)
$$

where $\sigma_{d}$ is the dissociation entropy of the two strands (the only concentration-dependent quantity in these expressions) and $T$ is in units of energy $(k=1)$ The second sum starts from $n=0$ (instead of from $n=n_{0}$ ) because this simply includes more (high-energy, i.e., improbable) states in the partition sum, but it introduces no additional complication and represents a better approximation for $n=n_{0}$ (see below). The energy $E_{1}(n), E_{2}(n)$ has a general shape similar to the graph of Fig. 3(a). We approximate it with a heuristic expression:

$$
E_{1}(n)=\alpha\left(n_{0}-n\right)^{1 / 2} \quad\left(\text { for } n \leq n_{0}\right)
$$

The exponent $1 / 2$ leads to good fits of the melting curves, and $n_{0}$ in (7) is the same as $n_{0}$ in (5) in order to minimize the number of parameters. The physical meaning of the heuristic form (7) is that in the kinked state the elastic energy is mostly in the ds part of the molecule, and thus given essentially by the upper form in (3). With the appropriate value for $n_{0}$, (7) is indeed an approximation for $E_{\mathrm{d}}\left(x_{\mathrm{eq}}\right)$ in (3) (upper branch), when $x_{\mathrm{eq}}$ is expressed in terms of $\left(N_{\mathrm{s}}+n\right)$, as we show later. For the energy $E_{2}(n)$, we take the approximation that, in this regime (no kink), the ds part of the molecule is essentially straight (true for short molecules) and the elastic energy is mostly in the stretched ss part. We therefore write the stretching energy of a ss DNA molecule of $\left(N_{s}+n\right)$ bases held at an EED $x=\left(N_{\mathrm{d}}-n\right) a$, which is the contour length of the ds part (and $a \approx 0.33 \mathrm{~nm}$ is the contour length of ds DNA per bp):

$$
E_{2}(n)=\frac{9 T a^{2}}{4 l_{\mathrm{s}}^{2}} \frac{\left(N_{\mathrm{d}}-n\right)^{2}}{N_{\mathrm{s}}+n} .
$$

$l_{\mathrm{s}}=0.8 \mathrm{~nm}$ is the persistence length of ss DNA [27], the above energy being written in the quadratic approximation.

We can now describe the three different cases (circular, nicked, and linear molecule) with this basic model. For the nicked molecule, the elastic energy of the kinked state is $E_{1}(n)$ given in (7). For the circular molecule, the elastic energy of the kinked state contains the additional cost of forming a 3 bp bubble at the kink [16], i.e.,

$$
E_{1}^{\text {circ }}(n)=\alpha^{\prime}\left(n_{0}^{\prime}-n\right)^{1 / 2}+3(\epsilon-\sigma T) \quad\left(\text { for } n \leq n_{0}^{\prime}\right) .
$$

In both cases, the elastic energy of the smoothly bent state is $E_{2}$ given in (8). For the linear molecule, $E_{2}=$ $E_{1}=0$. The partition sums and average number of open bp $\langle n\rangle$ at temperature $T$ are finally as follows.

For the nicked molecules: 


$$
\begin{aligned}
\langle n\rangle_{\text {nick }}= & \left\{\sum_{n=0}^{n_{0}} n(n+1) \exp \left[-\frac{n(\epsilon-\sigma T)}{T}\right] \exp \left[-\frac{\alpha\left(n_{0}-n\right)^{1 / 2}}{T}\right]+\sum_{n=0}^{N_{\mathrm{d}}-1} n(n+1) \exp \left[-\frac{n(\epsilon-\sigma T)}{T}\right]\right. \\
& \left.\times \exp \left[-\frac{9 a^{2}\left(N_{\mathrm{d}}-n\right)^{2}}{4 l_{\mathrm{s}}^{2}\left(N_{\mathrm{s}}+n\right)}\right]+N_{\mathrm{d}} \exp \left[-\frac{N_{\mathrm{d}}(\epsilon-\sigma T)}{T}\right] \exp \left(\sigma_{\mathrm{d}}\right)\right\} \frac{1}{Z_{\text {nick }}}, \\
Z_{\text {nick }}= & \sum_{n=0}^{n_{0}}(n+1) \exp \left[-\frac{n(\epsilon-\sigma T)}{T}\right] \exp \left[-\frac{\alpha\left(n_{0}-n\right)^{1 / 2}}{T}\right]+\sum_{n=0}^{N_{\mathrm{d}}-1}(n+1) \exp \left[-\frac{n(\epsilon-\sigma T)}{T}\right] \\
& \times \exp \left[-\frac{9 a^{2}\left(N_{\mathrm{d}}-n\right)^{2}}{4 l_{\mathrm{s}}^{2}\left(N_{\mathrm{s}}+n\right)}\right]+\exp \left[-\frac{N_{\mathrm{d}}(\epsilon-\sigma T)}{T}\right] \exp \left(\sigma_{\mathrm{d}}\right) .
\end{aligned}
$$

For the circular molecules:

$$
\begin{aligned}
\langle n\rangle_{\text {circ }}= & \left\{\sum_{n=0}^{n_{0}^{\prime}}(n+3)(n+1) \exp \left[-\frac{(n+3)(\epsilon-\sigma T)}{T}\right] \exp \left[-\frac{\alpha^{\prime}\left(n_{0}^{\prime}-n\right)^{1 / 2}}{T}\right]+\sum_{n=0}^{N_{\mathrm{d}}-1} n(n+1) \exp \left[-\frac{n(\epsilon-\sigma T)}{T}\right]\right. \\
& \left.\times \exp \left[-\frac{9 a^{2}\left(N_{\mathrm{d}}-n\right)^{2}}{4 l_{\mathrm{s}}^{2}\left(N_{\mathrm{s}}+n\right)}\right]+N_{\mathrm{d}} \exp \left[-\frac{N_{\mathrm{d}}(\epsilon-\sigma T)}{T}\right] \exp \left(\sigma_{\mathrm{d}}\right)\right\} \frac{1}{Z_{\text {circ }}} \\
Z_{\text {circ }}= & \sum_{n=0}^{n_{0}^{\prime}}(n+1) \exp \left[-\frac{(n+3)(\epsilon-\sigma T)}{T}\right] \exp \left[-\frac{\alpha^{\prime}\left(n_{0}^{\prime}-n\right)^{1 / 2}}{T}\right]+\sum_{n=0}^{N_{\mathrm{d}}-1}(n+1) \exp \left[-\frac{n(\epsilon-\sigma T)}{T}\right] \\
& \times \exp \left[-\frac{9 a^{2}\left(N_{\mathrm{d}}-n\right)^{2}}{4 l_{\mathrm{s}}^{2}\left(N_{\mathrm{s}}+n\right)}\right]+\exp \left[-\frac{N_{\mathrm{d}}(\epsilon-\sigma T)}{T}\right] \exp \left(\sigma_{\mathrm{d}}\right) .
\end{aligned}
$$

For the linear molecules:

$$
\begin{aligned}
\langle n\rangle_{\text {lin }} & =\left\{\sum_{n=0}^{N_{\mathrm{d}}-1} n(n+1) \exp \left[-\frac{n(\epsilon-\sigma T)}{T}\right]+N_{\mathrm{d}} \exp \left[-\frac{N_{\mathrm{d}}(\epsilon-\sigma T)}{T}\right] \exp \left(\sigma_{d}\right)\right\} \frac{1}{Z_{\text {lin }}}, \\
Z_{\text {lin }} & =\sum_{n=0}^{N_{\mathrm{d}}-1}(n+1) \exp \left[-\frac{n(\epsilon-\sigma T)}{T}\right]+\exp \left[-\frac{N_{\mathrm{d}}(\epsilon-\sigma T)}{T}\right] \exp \left(\sigma_{\mathrm{d}}\right) .
\end{aligned}
$$

\section{Fitting procedure}

Using the equations above, we fit the melting curves using the procedure below. For each set of three melting curves (linear, nicked, and circular molecule), we perform the following steps.

(1) Fit the melting curve of the linear molecule [using Eq. (12)], determining $\epsilon, \sigma, \sigma_{d}$.

(2) Use the value $n_{0}=26$ (the same for all nicked molecules); use the same $\epsilon, \sigma, \sigma_{d}$ determined in step (1), and Eq. (10) to fit the melting curve for the nicked molecule, determining $\alpha$.

(3) Use the value $n_{0}^{\prime}=19$ (the same for all circular molecules); use the same $\epsilon, \sigma, \sigma_{d}$ determined in step (1), and Eq. (11) to fit the melting curve for the circular molecule, determining $\alpha^{\prime}$.

The elastic energy extracted from the fits (in units of $T$, where $T=300 \mathrm{~K}$ is room temperature) is then

$$
E_{\mathrm{el}}=\alpha \sqrt{n_{0}} / 300
$$

for the nicked molecule, and

$$
E_{\mathrm{el}}^{\prime}=\alpha^{\prime} \sqrt{n_{0}^{\prime}} / 300+3(\epsilon / 300-\sigma)
$$

for the circular molecule.

To summarize, $\epsilon, \sigma, \sigma_{\mathrm{d}}$ are determined using the linear molecule, so that the fits for the nicked and circular molecule are reduced to one-parameter fits, which determine $\alpha$ and $\alpha^{\prime}$ and thus the elastic energies. The values of $n_{0}, n_{0}^{\prime}$ are chosen once and for all (the same for all molecules in this study) to produce the best overall fits for all the melting curves. We observe that the values of the energies obtained are robust with respect to changes in $n_{0}, n_{0}^{\prime}$ (if $n_{0}$ is changed, $\alpha$ changes, but $\alpha \sqrt{n_{0}}$ remains essentially the same).

There is, however, one difficulty. Step (1) above does not univocally determine $\epsilon$ and $\sigma$ : there is a range of values which give comparable fits. The reason is that, in the simple zipper model, the midpoint of the melting transition is essentially $T_{\mathrm{m}}=\epsilon / \sigma$, so varying $\epsilon$ and $\sigma$ (within a factor $\sim 1.5$ ) keeping the ratio fixed produces similar melting curves. Different $\epsilon, \sigma$ values in step (2) then produce a different value for $\alpha$ and thus the elastic energy; specifically, relatively small values for $\epsilon, \sigma$ produce relatively small values for $E_{\mathrm{el}}$. We overcome this problem using the 
independent measurements of the elastic energy of the nicked molecules obtained through the dimerization equilibrium method $[19,20]$. That is, we choose $\epsilon, \sigma$ in step (1) such that the elastic energy obtained in step (2) is consistent with the independent dimerization equilibrium measurements. Then, as explained above, we use these values to extract the unknown elastic energy $E_{\mathrm{el}}^{\prime}$ of the circular molecule.

The fits using the above functions are stable in the sense that the extracted elastic energy $E_{\mathrm{el}}^{\prime}$ does not change too much (within $\sim 0.5 \mathrm{~T}$ ) if we change parameter values $\left[n_{0}\right.$, $n_{0}^{\prime}$, the exponent $1 / 2$ in the expressions (7) and (9)] in a range compatible with obtaining reasonable fits, or if we add (for example) a loop-entropy term for the bubble in the first sum of Eq. (11), and other similar modifications that we explored.

\section{Analysis}

In Fig. 4(a) we show the melting curves and fits for the linear, nicked, and circular molecule with $N_{\mathrm{d}}=30, N_{\mathrm{s}}=$ 15. (The base sequence is given in Materials and Methods.) The parameter values are: $\epsilon=4309, \sigma=12.35, \sigma_{\mathrm{d}}=$ 5.63 for all three cases, and $\alpha=570 \pm 10$ for the nicked molecule, resulting in $E_{\mathrm{el}}=9.7 \pm 0.2 \mathrm{~T}$ (identical, by construction, to the result from the dimerization equilibrium measurement); and $\alpha^{\prime}=300 \pm 20$ for the circular molecule, resulting in $E_{\mathrm{el}}^{\prime}=10.3 \pm 0.3 \mathrm{~T}$. These values are the $N_{\mathrm{s}}=15$ data points in Fig. 5(b). The values of $\epsilon, \sigma, \sigma_{\mathrm{d}}$ are given above for completeness, but they are effective parameters only for this particular model and sequences. The error on the value of $\alpha$ (and thus $E_{\mathrm{el}}$ ) is the statistical error of the fit. These are also the error bars reported in Fig. 5. Systematic errors on the value of $E_{\mathrm{el}}$ due to the choice of model are harder to evaluate; by varying the details of the model, as mentioned above, and also by considering the absolute "calibration" obtained from the dimerization equilibrium measurements, we estimate that the systematic error on $E_{\mathrm{el}}$ is probably within $\pm 0.5 \mathrm{~T}$. In Fig. 4(b) we show an example of melting curves corresponding to one of the data points of Fig. 5(a), namely, $N_{\mathrm{d}}=41, N_{\mathrm{s}}=19$. Here the parameter values are $\epsilon=4233, \sigma=11.95, \sigma_{\mathrm{d}}=5.90, \alpha=586 \pm 8, \alpha^{\prime}=$ $349 \pm 11$, resulting in $E_{\mathrm{el}}=10.0 \pm 0.1 \mathrm{~T}, E_{\mathrm{el}}^{\prime}=11.6 \pm$ $0.2 \mathrm{~T}$.

The quality of the fits displayed in Fig. 4 may not seem very impressive, but it is in fact not bad. It is to be remembered that the circular and nicked curves are oneparameter fits (determining the elastic energy), and that the same approach is used for the 30 different melting curves in the study. The fits of the linear melting curves systematically underestimate the UV absorption signal in the low-temperature ("premelting") region: this is a deficiency of the zipper model approach, which fails to account for "fraying" of individual bp. The fits for the circular and nicked molecules capture (apart from the overall temperature shift) the main characteristic feature of these melting curves' shapes, which is a steeper rise before the midpoint compared to the linear curves' shapes, due to the release of the elastic energy, which drops abruptly for $n>n_{0}$ in the model [Fig. 3(c)] and in the real system [Fig. 3(a)]. We can now see, a posteriori, the significance of the values $n_{0}, n_{0}^{\prime} \sim 20$ which we found to produce good fits. Namely, in the kinked state, most of the elastic energy is in the ds part of the molecule, given essentially by $E_{\mathrm{d}}\left(x_{\mathrm{eq}}\right)$ in (3). For small $x_{\mathrm{eq}}$, this can be expressed analytically in terms of $\left(N_{\mathrm{s}}+n\right)$ by expanding Eq. (3) and using the first term in (13). The result is $E_{\mathrm{d}}(n) \approx E_{\mathrm{d}}(n=0)\left[1-\left(l_{\mathrm{s}}^{2} / \pi R^{2}\right)\left(\tau_{\mathrm{c}} / 9 T\right) n\right]$, while $(7)$ gives, for small $n$ : $E_{1}(n) \approx E_{1}(n=0)\left[1-n / 2 n_{0}\right]$. Comparing, we find $n_{0} \approx(\pi / 2)\left(R / l_{\mathrm{s}}\right)^{2}\left(9 T / \tau_{\mathrm{c}}\right)$. With our values $\tau_{\mathrm{c}} \approx 30 \mathrm{pN} \times \mathrm{nm} \approx 8 \mathrm{~T}, \quad R \approx L \approx 3 \mathrm{~nm}$ (for $N_{\mathrm{d}}=18$ ), this gives $n_{0} \approx 22$.

Referring to Fig. 1, the simplest way to explore the mechanical properties is to measure the elastic energy for a series of molecules with increasing $N_{\mathrm{s}}$, at fixed $N_{\mathrm{d}}$ : this has the effect of increasing the EED $x$. We followed this strategy in $[19,20]$ for the case of nicked DNA (i.e., with molecules formed by hybridizing two linear strands). However, in the non-nicked case, this would require preparing a series of circular strands of increasing length, which is cumbersome. Instead, here we keep the length of the circular strand fixed and vary the length of the complementary linear strand, i.e., we measure the elastic energy for a series of molecules with increasing $N_{\mathrm{s}}$, but with fixed $N_{\mathrm{s}}+N_{\mathrm{d}}=N_{\text {loop }}$. Figure 5(a) shows the results for $N_{\text {loop }}=60$, for the nicked and non-nicked (intact) molecules; Fig. 5(b) shows corresponding measurements for $N_{\text {loop }}=45$. As is obvious from the figure, the qualitative behavior is the same for the intact and the nicked molecules, namely, $E_{\text {tot }}$ is roughly linear in $N_{\mathrm{s}}$ in this regime, which, for the nicked molecules, corresponds to the kinked state as we have previously shown $[19,20]$. This suggests that the non-nicked molecules are also kinked in the regime shown, and that the kink is similarly described by a constant torque. We therefore assume that the formula (3) describes the bending elastic energy of the ds DNA for both the nicked and the intact case (with different values of the critical torque $\tau_{\mathrm{c}}$ ) and extract $\tau_{\mathrm{c}}$ in the two cases from the data of Fig. 5 using Eq. (3). We proceed as explained in the paragraph that follows Eq. (3), i.e., we calculate $E_{\text {tot }}$ from (4), using $E_{\mathrm{d}}(x)$ given by Eq. (3) and

$$
E_{\mathrm{s}}(x)=\frac{9 T}{4 N_{\mathrm{s}} l_{\mathrm{s}}^{2}}\left[x^{2}+\frac{x^{3}}{N_{\mathrm{s}} l_{\mathrm{s}}}+\frac{3 x^{4}}{\left(N_{\mathrm{s}} l_{\mathrm{s}}\right)^{2}}\right],
$$

which is a polynomial expansion of the Marko-Siggia expression [21]. The lines in Fig. 5(a) are computed in this way, and are seen to represent the data well with $\tau_{\mathrm{c}}=$ $27.0 \pm 0.7 \mathrm{pN} \times \mathrm{nm}$ for the nicked molecules and $\tau_{\mathrm{c}}=$ $31.3 \pm 0.6 \mathrm{pN} \times \mathrm{nm}$ for the non-nicked molecules. The error quoted reflects the range of $\tau_{\mathrm{c}}$ such that the calculated 
curve is, on average, within the error bars of the data points. A systematic error of $\pm 0.5 \mathrm{~T}$ in the values of $E_{\mathrm{el}}$ translates into a similar error for $E_{\text {tot }}$ and finally into an error $\pm 1.7 \mathrm{pN} \times \mathrm{nm}$ for $\tau_{\mathrm{c}}$. Similarly, the data of Fig. 5(b) (which represent molecules with a shorter loop and also a different base sequence around the position of the nick) give $\tau_{\mathrm{c}}=26.3 \pm 0.4 \mathrm{pN} \times \mathrm{nm}$ for the nicked case and $\tau_{\mathrm{c}}=28.8 \pm 0.5 \mathrm{pN} \times \mathrm{nm}$ for the non-nicked case. From these values we see the following: (1) If there is a sequence dependence of $\tau_{\mathrm{c}}$, it is not very prominent because the values obtained above with two different sequences are essentially within errors of each other; (2) The critical torque $\tau_{\mathrm{c}}$ does capture a materials property of the DNA, since measurements on two series of different molecules are well described by essentially the same $\tau_{\mathrm{c}}$. (For instance, for $N_{\mathrm{s}}=20$ in Fig. 5(a), the ds DNA has contour length $\sim 13 \mathrm{~nm}\left[N_{\mathrm{d}}=40\right]$, while for $N_{\mathrm{s}}=15$ in Fig. 5(b), the ds DNA has contour length $\left.\sim 10 \mathrm{~nm}\left[\mathrm{~N}_{\mathrm{d}}=30\right]\right)$. The fits in Fig. 5 are one-parameter fits $\left[\tau_{\mathrm{c}}\right.$ determining both the level and the slope of the plateaus in Fig. 5; see Eq. (3)]. That they capture the behavior of the measurements is evidence that the ds DNA is well described by the upper form of (3) in this regime, i.e., that the DNA is kinked. For comparison, we show in Fig. 5(c) $E_{\text {tot }}$ calculated for the case of Fig. 5(a) $\left(N_{\text {loop }}=60\right)$, assuming the ds DNA bending energy follows the WLC form (1). Clearly the result is incompatible with the measurements. On the other hand, the range of the experimental measurements (vs $N_{\mathrm{s}}$ ) is narrower than one would like (especially for $N_{\text {loop }}=$ 45). The reason is as follows: We do not choose $N_{\mathrm{s}}$ too small because we do not know the form of the stretching energy for very short ss DNA; the persistence length of ss DNA is $\sim 3$ bases, and for (13) to hold we need presumably several persistence lengths. At the other end, as we increase $N_{\mathrm{s}}$, the melting curves for the linear and circular molecules eventually become very close in temperature, i.e., the experimental signal (based on the melting curves) is small, and we feel the model (5) is no longer adequate to extract quantitative measurements.

\section{DISCUSSION}

In this study we characterize the nonlinear bending elasticity of short ds DNA molecules. The measured elastic energies for sharp bending are quantitatively reproduced by assuming a kinked state of the DNA in this regime, with a constant (independent of EED $x$ ) torque $\tau_{\mathrm{c}}$ at the kink, i.e., the elastic energy is linear in the kink angle, as was suggested in [11]. The same conclusion was arrived at for the case of nicked DNA [20]. The critical torque measured for the non-nicked sequences of Fig. $5(\mathrm{a}), \tau_{\mathrm{c}}=31.3 \mathrm{pN} \times$ $\mathrm{nm}$, is only slightly larger than the critical torque for the same sequences with a nick, which is $\tau_{\mathrm{c}}=27.0 \mathrm{pN} \times \mathrm{nm}$. In terms of energy, the elastic energy of the kinked, intact molecule is only $\sim 2 \mathrm{~T}$ larger than the energy of the corresponding nicked molecule [Fig. 5(a)]. This is consistent with previous estimates of the effect of a nick [28] and also consistent with the picture of bubble formation at the kink [16]. Namely, the difference in the freeenergy cost of forming the bubble for the non-nicked and nicked cases is essentially the loop entropy, which is $\sim 2 \mathrm{~T}$ [16,29].

For the thin-rod approximation considered here (which is reasonable if the boundary conditions allow free torsional degrees of freedom), the local torque is $\tau=B / R$, where $R$ is the local radius of curvature. Thus the critical torque $\tau_{\mathrm{c}}$ corresponds to a critical local radius of curvature $R_{\mathrm{c}}=B / \tau_{\mathrm{c}}=\left(T / \tau_{\mathrm{c}}\right) l_{\mathrm{p}} \approx 6 \mathrm{~nm} \quad$ using $\tau_{\mathrm{c}}=32 \mathrm{pN} \times$ $\mathrm{nm} \approx 8 \mathrm{~T}$. Writing the range of validity of the WLC energy (1) as $2 \pi R>C l_{\mathrm{p}}$ (see Sec. I), this corresponds to $C=2 \pi\left(T / \tau_{\mathrm{c}}\right) \approx 0.7$, which is actually exactly the original suggestion of Cloutier and Widom [12]. However, for a quantitative comparison, we should really calculate the $j$ factors using the bending energy (3). This is a nontrivial calculation that we propose to leave as an exercise for the reader. Similarly, the minicircles result [14], which at face value says $C \approx 0.5$, could also very well be consistent with our value of $\tau_{\mathrm{c}}$. This is because the critical radius $R_{\mathrm{c}}$ (defined as counter length $/ 2 \pi$ ) at which a minicircle becomes unstable to kinking is, presumably, not simply $R_{\mathrm{c}}=$ $B / \tau_{\mathrm{c}}$. Instead, this is a rather more complicated problem because of the different boundary conditions. For instance: torsional energy must in general be included [14]; the first instability as $R$ is reduced might not even be kinking, but out-of-plane bending; further, thermal fluctuations may be important in determining the threshold for kinking, whereas the above estimate is purely mechanical. Again, we propose to leave the quantitative connection between $\tau_{\mathrm{c}}$ and the minicircles result [14] as an open problem.

Figure 6 makes it clear that, for short molecules, the nonlinear (i.e., kinked) regime of bending is actually the dominant regime in the space of EED (this would not be the case if the critical torque were much larger). Correspondingly, using the elastic energy (1) for small EED (continuing the smoothly bent branch of the elastic energy in Fig. 6 for small $x$ ) results in a large overestimate of the elastic energy. In contrast, it is evident that the critical torque $\tau_{\mathrm{c}}$ introduces a universal energy scale $(\pi / 2) \tau_{\mathrm{c}} \approx 12 \mathrm{~T}$ in the physics of DNA bending (this is $\lim _{x \rightarrow 0} E_{\mathrm{d}}(x)$ in (3); see Fig. 6), a fact of some consequence in the molecular-biology processes associated with DNA bending. We refer to this quantity as an "energy scale" because it is a local quantity, independent of the length of the molecule; in contrast, there is no such scale for the WLC energy (1). In more structural terms, this energy scale is also essentially the cost of forming a localized ss bubble in the absence of external stresses [16] and must also be related to the energy cost of untwisting one turn of the double helix.

Equation (3) was shown to describe the measurements well in the kinked regime (Fig. 5); in the case of nicked 


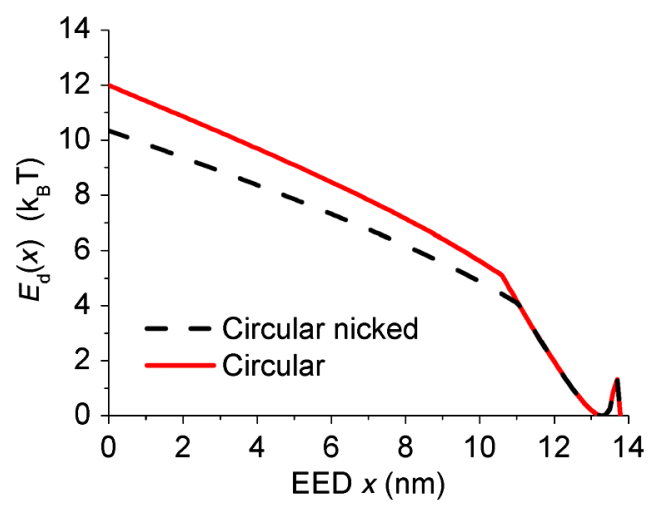

FIG. 6. Graph of the ds DNA bending energy $E_{\mathrm{d}}(x)$ vs EED $x$ from Eq. (3), for $N_{\mathrm{d}}=41$ (contour length $2 L=13.7 \mathrm{~nm}$ ). The solid line is the plot for $\tau_{\mathrm{c}}=31.3 \mathrm{pN} \times \mathrm{nm}$ (corresponding to the intact DNA); the dashed line is $\tau_{\mathrm{c}}=27.0 \mathrm{pN} \times \mathrm{nm}$ (corresponding to the nicked DNA); $B=50 \mathrm{kT} \times \mathrm{nm}$ in both cases. The zero-force EED is slightly smaller than the contour length $2 L$ because of thermal fluctuations [included in Eq. (3)]; the WLC energy (steep part of the curve, for $0 \leq E_{\mathrm{d}} \leq 5 \mathrm{kT}$, approximately) is approximately linear in EED $x$; the kinked state energy ( $5 \mathrm{kT} \leq E_{d} \leq 12 \mathrm{kT}$, approximately) is also approximately linear in EED $x$, but with a smaller slope. For these short molecules, most of the parameter space (in $x$ ) corresponds to the nonlinear elasticity (kinked) regime.

DNA, we know experimentally from the dimerization equilibrium measurements that (3) also gives a good description around the transition to the smoothly bent state and beyond [Fig. 3(a)] [20]. For non-nicked DNA, it proved difficult to obtain measurements in the transition region because the melting curves of the linear and circular molecules become very close, and the model (5) seems inadequate to draw quantitative conclusions. As a general remark, there is at least one effect missing from the theory (3) which may be important for non-nicked DNA around the transition region, namely, the contribution to the elastic (free) energy of allowing the kink to move. This has been considered before [30], but it was not introduced in the description (3) for simplicity; on the other hand, for nicked DNA, it seems reasonable to assume that the kink is pinned at the nick, so this effect is probably unimportant.

The model in Eq. (5) used to analyze the melting profiles has no pretense at physical insight and is merely a datafitting tool; however, the melting of mechanically stressed DNA in various configurations is in itself an interesting problem [31], and the experimental melting curves reported here may be valuable to those interested in this problem. A related question is the temperature dependence of the elastic parameters $B$ and $\tau_{\mathrm{c}}$ in the "premelting" region. (At the melting transition, it is doubtful whether the elastic energy of the molecule shown in Fig. 1 can be usefully described in terms of temperature-dependent elastic constants.) Recent measurements by the Vologodskii group [32] report that the persistence length $l_{\mathrm{p}}$ of $\mathrm{ds}$ DNA decreases by about $30 \%$ from $5^{\circ} \mathrm{C}$ to $60^{\circ} \mathrm{C}$, which corresponds to a roughly $15 \%$ decrease in $B=k T l_{\mathrm{p}}$. The present experiment is unsuitable to measure these effects [and they are not included in the analysis; for instance, $\alpha$ in Eq. (7) is taken as constant independent of temperature]. However, the temperature dependence of $\tau_{\mathrm{c}}$ can be measured in the future using the monomer-dimer equilibrium method.

In conclusion, we show that two materials parametersthe bending modulus $B$ and the newly introduced critical torque $\tau_{\mathrm{c}}$-largely suffice for a quantitative description of DNA bending elasticity in the linear and nonlinear regimes.

\section{ACKNOWLEDGMENTS}

This work was supported by the UC Lab Research Program and by NSF Grant No. DMR-1006162.

[1] P. J. Flory, Principles of Polymer Chemistry (Cornell University Press, Ithaca, 1953).

[2] J. Monod, J-P. Changeux, and F. Jacob, Allosteric Proteins and Cellular Control Systems, J. Mol. Biol. 6, 306 (1963).

[3] M. F. Perutz, Nature of Haem-Haem Interaction, Nature (London) 237, 495 (1972).

[4] J. J. Hopfield, Relation between Structure, Co-operativity and Spectra in a Model of Hemoglobin Action, J. Mol. Biol. 77, 207 (1973).

[5] Y. Savir and T. Tlusty, Conformational Proofreading: The Impact of Conformational Changes on the Specificity of Molecular Recognition, PLoS ONE 2, e468 (2007).

[6] C-Y. Tseng, A. Wang, and G. Zocchi, Mechano-Chemistry of the Enzyme Guanylate Kinase, Europhys. Lett. 91, 18005 (2010).

[7] G. Zocchi, Controlling Proteins through Molecular Springs, Annu. Rev. Biophys. 38, 75 (2009).

[8] G. H. Garcia, P. Grayson, L. Han, M. Inamdar, J. Kondev, P. C. Nelson, R. Phillips, J. Widom, and P. A. Wiggins, Biological Consequences of Tightly Bent DNA: The Other Life of a Macromolecular Celebrity, Biopolymers 85, 115 (2006).

[9] N. B. Becker and R. Everaers, DNA Nanomechanics in the Nucleosome, Structure 17, 579 (2009).

[10] C. Bustamante, Z. Bryant, and S. B. Smith, Ten Years of Tension: Single-Molecule DNA Mechanics, Nature (London) 421, 423 (2003).

[11] P. A. Wiggins, T. van der Heijden, F. Moreno-Herrero, A. Spakowitz, R. Phillips, J. Widom, C. Dekker, and P. C. Nelson, High Flexibility of DNA on Short Length Scales Probed by Atomic Force Microscopy, Nature Nanotech. 1, 137 (2006).

[12] T. E. Cloutier and J. Widom, Spontaneous Sharp Bending of Double-Stranded DNA, Mol. Cell 14, 355 (2004).

[13] Q. Du, C. Smith, N. Shiffeldrim, M. Vologodskaia, and A. Vologodskii, Cyclization of Short DNA Fragments and Bending Fluctuations of the Double Helix, Proc. Natl. Acad. Sci. U.S.A. 102, 5397 (2005). 
[14] Q. Du, A. Kotlyar, and A. Vologodskii, Kinking the Double Helix by Bending Deformation, Nucleic Acids Res. 36, 1120 (2007).

[15] C. Yuan, H. Chen, X. W. Lou, and L. A. Archer, DNA Bending Stiffness on Small Length Scales, Phys. Rev. Lett. 100, 018102 (2008).

[16] J. Yan and J. F. Marko, Localized Single-Stranded Bubble Mechanism for Cyclization of Short Double Helix DNA, Phys. Rev. Lett. 93, 108108 (2004).

[17] B. Choi, G. Zocchi, Y. Wu, S. Chan, and L.J. Perry, Allosteric Control through Mechanical Tension, Phys. Rev. Lett. 95, 078102 (2005).

[18] A. Wang and G. Zocchi, Elastic Energy Driven Polymerization, Biophys. J. 96, 2344 (2009).

[19] H. Qu, C-Y. Tseng, Y. Wang, A. J. Levine, and G. Zocchi, The Elastic Energy of Sharply Bent Nicked DNA, Europhys. Lett. 90, 18003 (2010).

[20] H. Qu and G. Zocchi, The Complete Bending Energy Function for Nicked DNA, Europhys. Lett. 94, 18003 (2011).

[21] C. Bustamante, J. F. Marko, E. D. Siggia, and S. Smith, Entropic Elasticity of $\lambda$-Phage DNA, Science 265, 1599 (1994).

[22] V. Ivanov, Y. Zeng, and G. Zocchi, Statistical Mechanics of Base Stacking and Pairing in DNA Melting, Phys. Rev. E 70, 051907 (2004).

[23] N. R. Markham and M. Zuker, DINAMelt Web Server for Nucleic Acid Melting Prediction, Nucleic Acids Res. 33, W577 (2005).
[24] C. Kittel, Phase Transition of a Molecular Zipper, Am. J. Phys. 37, 917 (1969).

[25] M. Peyrard, S. Cuesta-López, and G. James, Modelling DNA at the Mesoscale: A Challenge for Nonlinear Science?, Nonlinearity 21, T91 (2008).

[26] S. Ares, N. K. Voulgarakis, K.Ø. Rasmussen, and A. R. Bishop, Bubble Nucleation and Cooperativity in DNA Melting, Phys. Rev. Lett. 94, 035504 (2005).

[27] S. B. Smith, Y. Cui, and C. Bustamante, Overstretching BDNA: The Elastic Response of Individual DoubleStranded and Single-Stranded DNA Molecules, Science 271, 795 (1996).

[28] Y. Zhang and D. M. Crothers, High-Throughput Approach for Detection of DNA Bending and Flexibility Based on Cyclization, Proc. Natl. Acad. Sci. U.S.A. 100, 3161 (2003).

[29] D. H. Mathews, J. Sabina, M. Zuker, and D. H. Turner, Expanded Sequence Dependence of Thermodynamic Parameters Improves Prediction of RNA Secondary Structure, J. Mol. Biol. 288, 911 (1999).

[30] P. A. Wiggins, R. Phillips, and P. C. Nelson, Exact Theory of Kinkable Elastic Polymers, Phys. Rev. E 71, 021909 (2005).

[31] D. R. Nelson, Statistical Physics of Unzipping DNA, arXiv:cond-mat/0309559v1.

[32] S. Geggier, A. Kotlyar, and A. Vologodskii, Temperature Dependence of DNA Persistence Length, Nucleic Acids Res. 39, 1419 (2010). 